\title{
Task Space Approach of Robust Nonlinear Control for a 6 DOF Parallel Manipulator
}

\author{
Hag Seong Kim \\ Agency for Defense Development
}

Korea

\section{Introduction}

The dynamics and kinematics of a parallel manipulator has been widely researched by virtue of its a high force-to-weight ratio and widespread applications ranging from vehicle or flight simulator to machine tool despite a smaller workspace than a serial robot system (Merlet, 2000). Such a parallel system has been paid special attention as a typical multi-input multi-output nonlinear system to retain a high control performance. A control scheme for a 6 DOF parallel manipulator can be classified into two groups: a joint space based control scheme (Honegger et al., 2000; Kang et al., 1996; Kim et al., 2000; Nguyen et al., 1993; Sirouspour \& Salcudean, 2001) and a task space based control strategy (Kang et al., 1996; Park, 1999; Ting et al., 1999). It is easy to realize the joint space based control scheme to a parallel manipulator as if the decoupled single-input single-output (SISO) control systems activate for a parallel mechanism. The simplicity has let many research activities pursue more specific approaches. As a result, the novel joint space based control approaches have been studied to improve the control performance by rejecting the nonlinear effects in the equations of motion (Honegger et al., 2000; Kang et al., 1996; Kim et al., 2000; Nguyen et al., 1993; Sirouspour \& Salcudean, 2001). Particularly, for a parallel system driven by a hydraulic-servo system, joint space based robust nonlinear control scheme (Kim et al., 2000) has proposed. However, the research has dealt with excessively conservative uncertainties including gravity and known dynamic characteristics even though the friction effect can be neglected by the hydrostatic bearing. On the other hand, a task space based control for a 6 DOF parallel manipulator has a potential to meet excellent control performances under system uncertainties: inertia, modeling error, friction, etc. However, its scheme may be realized by the obtained the 6 DOF system state through a costly sensor or a novel nonlinear state estimation methodology. $H_{\infty}$ robust control strategy (Park, 1999) and the adaptive

control scheme (Ting et al., 1999) have been studied as the examples of task space based control. However, there have been still some weak points in the previous researches; the linearized model based approach and a simulation study only, respectively. Another task if space based nonlinear control scheme has been proposed to a Stewart platform (Kang et al., 1996). However, it has also shown the computational simulation results only on the assumption to the system uncertainties that seems excessive. Furthermore, its treatment on stick-slip friction is minimal, which may give rise to serious deterioration of control performance in a real system where the frictional property is not negligible.

Source: Parallel Manipulators, New Developments, Book edited by: Jee-Hwan Ryu, ISBN 978-3-902613-20-2, pp. 498, April 2008, I-Tech Education and Publishing, Vienna, Austria 
This paper focus on a theoretical and experimental study to develop a task space based robust nonlinear controller for a 6 DOF parallel system. This study starts from the indirect estimation of the system state essential to the task space based control instead of the application of a costly 6 DOF sensor. The 6 DOF system state is obtained by a numerical forward kinematic solution based on the Newton-Raphson method (Dieudonne et al., 1972; Nguyen et al., 1993) and an alpha-beta tracker (Friedland, 1973; Lewis, 1986). The feasibility of the indirect state estimation method is confirmed by the comparison of the results from the alpha-beta tracker with forward kinematic solution and the measured gyro signals, respectively. Then, the Friedland-Park friction estimator (Friedland \& Park, 1992) is employed to attenuate the frictional disturbances in the actuators. The friction estimates are also compared to independently measured friction values (Park, 1999), which show reasonable agreement. Finally, the task space based robust nonlinear control scheme with the proposed estimation methods for system state and friction is proposed and theoretically proved by the representation of the practical stability for a 6 DOF parallel manipulator with uncertainties such as inertia, modeling error, friction, and measurement errors, etc. The proposed controller law exhibits remarkable regulation and tracking control performances to given several inputs. It is also shown that the proposed robust nonlinear control law with task space approach outperforms the task space based nonlinear control without the additional input for a robust control and a PID controller with the two independent estimators for the system state and friction in joint space.

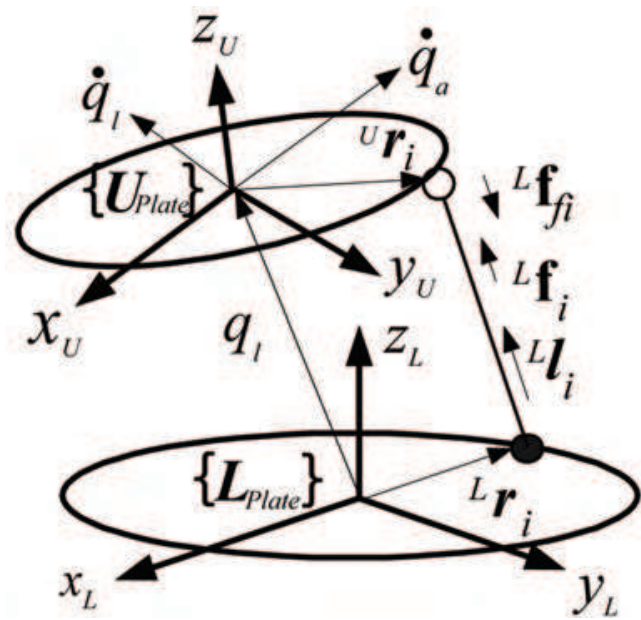

Fig. 1. The vector definitions for the mathematical model of the 6 DOF parallel manipulator

\section{Dynamic model of a 6 DOF parallel manipulator}

This section briefly describes the dynamic model of a 6 DOF parallel manipulator that has been extensively studied (Dasgupta \& Mruthyunjaya, 1998; Kang et al., 1996; Kim et al., 2000). Fig. 1 describes the $\left\{L_{\text {plate }}\right\}$ and $\left\{U_{\text {plate }}\right\}$ coordinate systems as the base coordinate system for the inertial frame and the moving coordinate system for the body-fixed frame, respectively. Linear motions along the $x_{L}-y_{L}-z_{L}$ axis are surge $(x)$, sway $(y)$, and heave 
$(z)$, respectively. Rotational motions corresponding to the $x_{U}-y_{U}-z_{U}$ Euler angles are roll $\left(\theta_{r}\right)$, pitch $\left(\theta_{p}\right)$, and yaw $\left(\theta_{y}\right)$, respectively. The definition of each vector required to derive the kinematic and dynamic equations of the parallel mechanism are depicted in Fig. 1 $(i=1$ to 6$)$ as well.

For the angular and linear motions of the parallel manipulator, the following dynamic model can be derived by the Euler-Lagrangian method (Kang et al., 1996; Kim et al., 2000)

$$
\mathbf{M}\left(\mathbf{q}_{u}, \xi\right) \ddot{\mathbf{q}}_{u}+\mathbf{C}\left(\mathbf{q}_{u}, \dot{\mathbf{q}}_{u}, \xi\right) \dot{\mathbf{q}}_{u}+\mathbf{G}\left(\mathbf{q}_{u}, \xi\right)=\mathbf{J}^{T}\left(\mathbf{q}_{u}\right)\left(\mathbf{f}-\mathbf{f}_{f}\right),
$$

where $\mathbf{M}(\cdot) \in \mathbf{R}^{6 \times 6}$ is inertia, $\mathbf{C}(\cdot) \in \mathbf{R}^{6 \times 6}$ is Coriolis and centrifugal force, $\mathbf{G}(\cdot) \in \mathbf{R}^{6}$ is gravitational force, $\mathbf{J}(\cdot) \in \mathbf{R}^{6 \times 6}$ is Jacobian, $\xi$ denotes uncertainties, $\mathbf{q}_{u}=\left[\begin{array}{ll}\mathbf{q}_{l}{ }^{T} & \mathbf{q}_{a}{ }^{T}\end{array}\right]^{T} \in \mathbf{R}^{6}$, $\mathbf{q}_{l}=\left[\begin{array}{lll}x & y & z\end{array}\right]^{T}, \quad \mathbf{q}_{a}=\left[\begin{array}{lll}\theta_{r} & \theta_{p} & \theta_{y}\end{array}\right]^{T}, \mathbf{f} \in \mathbf{R}^{6}$ denotes the actuator forces, and $\mathbf{f}_{f} \in \mathbf{R}^{6}$ is an equivalent friction vector for actuators and joints.

In (1), it is assumed that $\dot{\mathbf{M}}-2 \mathbf{C}$ satisfies the skew symmetric property (Spong \& Vidyasagar, 1989) and the parallel system is mechanically designed to avoid singularity of Jacobian matrix in the workspace. It is further assumed that system uncertainties are closed and bounded. The above assumptions are summarized as

Assumption 1. The Jacobian is not singular.

Assumption 2. If $\xi$ (constant or time-varying) represents uncertainties that include inertia, modeling error, and measurement noise, $\xi \in \Xi$, where $\Xi$ is compact set.

The actuator dynamics (both electrical and mechanical) may be neglected in this system to simplify the system model and apply the robust nonlinear control theory with ease. Nevertheless, in this paper, the friction of each actuator is considered since the friction may be the primary cause that deteriorates a control performance.

\section{Estimations of system state and friction}

This section briefly describes both the indirect system state estimation methodology and friction estimator. The length of each cylinder may be readily measured by relatively cheap sensor and directly applied to the joint space based control scheme, while the task space based control scheme requires 6 DOF data information which may be extracted by costly sensor. Alternatively, nonlinear observer (Kang, et al., 1998) may be implemented to acquire the 6 DOF system state. However, the idea is not adopted since the overall system stability and control performance may not be guaranteed on the observed state in a short time that can be appeared by undesirable condition called "peaking phenomenon" in a nonlinear system (Khalil, 1996). Furthermore, the mathematical relation between the angular velocities of the upper plate and the linear velocities of actuators (Dieudonne et al., 1972; Honegger et al., 2000; Kim et al., 2000; Nguyen et al., 1993) may be applied to calculate the angular velocities of the rigid upper plate. However, the mathematical relation is somewhat complicated, and can levy much computational time on the control system. Therefore, the following indirect state estimation methodology is presented to surmount such an adverse circumstance. The 6 DOF system state is estimated with the Newton-Raphson method and an alpha-beta tracker. The Newton-Raphson method performs well with a proper choice of the initial condition (Dieudonne et al., 1972). Furthermore, the derivatives of the system states are easily calculated via an alpha-beta tracker even though the tracker is applicable to 
a system with acceleration of zero mean process noise (Friedland, 1973; Lewis, 1986). In addition to that, the proposed control scheme in this paper also needs the first/second order derivatives of the arbitrary, continuous, desired position information, which cannot be precomputed. Therefore, the indirect method is available to yield derivatives of arbitrary inputs as well.

This section also considers the equivalent friction estimator in order to reject the undesirable friction property. In general, the frictional property is changeable in the various conditions like lubrication, load, and even time, which means there may be uncertainties in friction. Furthermore, the uncertain and excessive feed-forward compensation may result in a phenomenon like limit cycle or undesirable control performance. Therefore, the FriedlandPark friction observer is pursued as a framework to compensate stick-slip friction among the previous approaches (Amstrong-Hélouvry et al., 1994; Panteley et al., 1998) since the Friedland-Park friction observer has shown excellent observer performance against general friction properties in spite of consideration on ideal Coulomb friction model (Friedland \& Park, 1992). Unfortunately, the friction observer cannot be directly applied to the parallel mechanism due to its highly coupled nonlinear dynamics since it is targeted to SISO system. Therefore, the equivalent friction estimator is applied only in the context of friction estimator and control design with the assumption that each actuator system of the parallel manipulator could be modeled as an equivalent SISO system. The uncertainties in the friction estimates are regarded as an element of system uncertainties, which will be discussed later in the control design section. The observer with a readily implemented structure is briefly described for a decoupled parallel system as in a equivalent SISO system in the following.

$$
\ddot{x}_{f}=\dot{v}_{f}=\frac{1}{m_{e q}}\left(w_{f}-f_{f}\right), f_{f}=c_{f} \cdot \operatorname{sgn}\left(v_{f}\right),
$$

where $x_{f}$ and $v_{f}$ are the estimated linear displacement, velocity of each actuator, respectively, $m_{e q}$ is the equivalent load of each cylinder, $f_{f}$ is the actuator friction of each cylinder, $c_{f}$ is the friction parameter, and $w_{f}$ is the control force that includes additional robust control and estimated friction terms of each actuator. Then, the parameter $\hat{c}_{f}$ can be updated by

$$
\begin{gathered}
\hat{c}_{f}=z_{f}-k_{f}\left|v_{f}\right|^{\mu_{f}}, \\
\dot{z}_{f}=\frac{1}{m_{e q}} k_{f} \mu_{f}\left|v_{f}\right|^{\mu_{f}-1}\left(w_{f}-\hat{f}_{f}\right) \operatorname{sgn}\left(v_{f}\right),
\end{gathered}
$$

where $\hat{c}_{f}$ is estimated friction parameter, $z_{f}$ is variable, $k_{f}>0$ and $\mu_{f} \geq 1$ are constant gains. It should be noted that $\mu_{f} \geq 1$ since the dynamics of the variable $z_{f}$ in (4) cannot be defined at the zero velocity in the case of $\mu_{f}<1$.

It should be noted that the indirect state estimation scheme and the friction estimator have not been widely applied to a 6 DOF parallel system even though these may be often used independently in practice. 


\section{Robust nonlinear control design}

This section presents the design of the robust nonlinear controller and the accompanying stability analysis for the 6 DOF parallel manipulator control system equipped with the aforementioned estimators in the previous section. The robust nonlinear control theory has been researched widely to guarantee practical stability for a nonlinear system with the detailed definitions (practical stability, uniform ultimate boundedness, etc.) and the assumptions described in Barmish et al.(1983), Corless \& Leitmann (1981), and Khalil (1996). However, it may not be straightforward applied to a nonlinear system with stick-slip friction that does not satisfy the Caratheodory condition (Corless \& Leitmann, 1981) at zero velocity where the friction represented by set-value map (Caratheodory condition is mathematically required to guarantee the existence and the continuity of the solution). Nevertheless, the previous studies (Hahn, 1967; Radcliffe \& Southward, 1990) have shown that there still exists a continuous solution under stick-slip friction from a practical viewpoint. Therefore, it may be not too impudent to suppose the existence of a solution to a mathematical model for a real system with friction, which makes it possible to apply a robust nonlinear control theory into a parallel system with stick-slip friction. The following assumptions 3 is additionally made for a robust control design.

Assumption 3. There exist positive constant $\sigma_{\bar{M}}, \sigma_{\underline{M}}>0$ such that

$$
\sigma_{\underline{M}} \mathbf{I}<\mathbf{M}\left(\mathbf{q}_{\mathrm{U}}, \xi\right)<\sigma_{\bar{M}} \mathbf{I},
$$

where $\forall \mathbf{q}_{u} \in \mathbf{D}_{q r}, \mathbf{D}_{q r}=\left\{\mathbf{q}_{u} \mid\left\|\mathbf{q}_{u}\right\| \leq r, \quad r \in[0, \infty)\right\}, \xi \in \Xi$, and $\Xi$ is compact.

If the measurements or estimates of $6 \mathrm{DOF}$ positional data contain uncertainties, the control function with the inverse of $\mathbf{J}^{T}(\cdot)$ is no longer valid. In this case, the proposed robust nonlinear control strategy requires additional assumptions:

Assumption 4. There exist a constant $k_{1}$ such that

$$
\left\|\delta \mathbf{J}^{T} \mathbf{J}_{e}^{-T}\right\| \leq k_{1}<1,
$$

where $\mathbf{J}_{e}{ }^{T}\left(\mathbf{q}_{u}^{\prime}, \xi\right)=\mathbf{J}^{T}\left(\mathbf{q}_{u}, 0\right)+\delta \mathbf{J}^{T}\left(\delta \mathbf{q}_{u}, \xi\right), \mathbf{q}_{u}^{\prime}=\mathbf{q}_{u}-\delta \mathbf{q}_{u}, \mathbf{q}_{u}^{\prime}$ is a vector of 6 DOF estimated system state, and $\delta \mathbf{q}_{u}$ is a vector of uncertainties in the 6 DOF positional estimated values.

Assumption 5. There exist a constant $k_{2}$ such that

$$
\left\|\delta \dot{\mathbf{q}}_{u}+\mathbf{S} \delta \mathbf{q}_{u}\right\| \leq k_{2},
$$

where $\delta \dot{\mathbf{q}}_{u}$ is a vector of uncertainties in the measured or estimated 6 DOF velocities, and

$$
\mathbf{S}=\operatorname{diag}\left(\mathbf{S}_{i}\right) \in \mathbf{R}^{6 \times 6}, \mathbf{S}_{i}>0 \text {. }
$$

Assumption 6. It is assumed that each matrix in (1) can be represented as nominal plus deviation:

$$
\begin{gathered}
\mathbf{M}\left(\mathbf{q}_{u}, \xi\right)=\mathbf{M}_{0}\left(\mathbf{q}_{u}^{\prime}, 0\right)+\delta \mathbf{M}\left(\mathbf{q}_{u}, \xi\right), \quad \mathbf{C}\left(\mathbf{q}_{u}, \dot{\mathbf{q}}_{u}, \xi\right)=\mathbf{C}_{0}\left(\mathbf{q}_{u}^{\prime}, \dot{\mathbf{q}}_{u}^{\prime}, 0\right)+\delta \mathbf{C}\left(\mathbf{q}_{u}, \dot{\mathbf{q}}_{u}, \xi\right), \\
\mathbf{G}\left(\mathbf{q}_{u}, \xi\right)=\mathbf{G}_{0}\left(\mathbf{q}_{u}^{\prime}, 0\right)+\delta \mathbf{G}\left(\mathbf{q}_{u}, \xi\right), \text { and } \mathbf{f}_{f}\left(\mathbf{q}_{u}, \dot{\mathbf{q}}_{u}, \xi\right)=\hat{\mathbf{f}}_{f}\left(\mathbf{q}_{u}^{\prime}, \dot{\mathbf{q}}_{u}^{\prime}, 0\right)+\delta \mathbf{f}_{f}\left(\mathbf{q}_{u}, \dot{\mathbf{q}}_{u}, \xi\right) .
\end{gathered}
$$

The excessive uncertainties in the control design (Kang et al., 1996; Kim et al., 2000) including the nominal values of gravitational force and Coriolis force may result in 
undesirable control performance. Therefore, in the proposed control strategy of this paper, the uncertainties are minimized by directly compensating for the nominal gravitational force, Coriolis force, etc. on the assumption 6. Furthermore, the uncertainties in friction estimates are also considered as the element of system uncertainties.

Remark 7. The existence of the constant $k_{1}$ and $k_{2}$ in assumptions 4 and 5 seem to be restrictive. If the uncertainties or errors in the measurements or estimates of 6 DOF data are large or cannot be bounded, it is impossible to apply a MIMO robust control scheme. If the 6 DOF positional data are made directly available via a 6 DOF sensor, $\delta \mathbf{q}_{u}$ is negligible in the assumptions 4-6. The experimental results based on the indirect state estimation methodology show the reasonable agreement of the estimates later in Section 5.1.

Theorem 8. Suppose that the system (1) satisfies the assumptions 1-6 with the definition of tracking error $\tilde{\mathbf{q}}_{u}=\mathbf{q}_{u}-\mathbf{q}_{u_{d}}$, where $\mathbf{q}_{u_{d}} \in \mathbf{R}^{6}$ is the desired trajectory. In addition, suppose that there exist the bounding functions $\rho_{1}(\cdot)$ and $\rho_{2}(\cdot)$ that satisfy the condition (9). Then, the system (1) is practically stable in the domain $\mathbf{D}_{r}=\left\{\mathbf{e} \in \mathbf{R}^{12} \mid\|\mathbf{e}\| \leq r, \quad \mathbf{e} \equiv\left[\begin{array}{ll}\tilde{\mathbf{q}}_{U}^{\prime} & \dot{\tilde{\mathbf{q}}}_{U}^{\prime}\end{array}\right]^{T}, r \in[0, \infty)\right\}$ for a given $\varepsilon$ with the robust nonlinear control law (10).

$$
\mathbf{f}_{e q}=\mathbf{J}_{e}^{-T}\left\{\mathbf{G}_{0}\left(\mathbf{q}_{u}^{\prime}\right)+\mathbf{M}_{0}\left(\mathbf{q}_{u}^{\prime}\right)\left(\ddot{\mathbf{q}}_{u_{d}}-\mathbf{S} \dot{\tilde{\mathbf{q}}}_{u}^{\prime}\right)+\mathbf{C}_{0}\left(\mathbf{q}_{u}^{\prime}, \dot{\mathbf{q}}_{u}^{\prime}\right)\left(\dot{\mathbf{q}}_{u_{d}}-\mathbf{S} \tilde{\mathbf{q}}_{u}^{\prime}\right)-\mathbf{K}_{p} \tilde{\mathbf{q}}_{u}^{\prime}-\mathbf{K}_{V} \dot{\tilde{\mathbf{q}}}_{U}^{\prime}+\mathbf{J}_{e}^{T}(\cdot) \hat{\mathbf{f}}_{f}\right\},
$$

where $\mathbf{K}_{p}, \mathbf{K}_{V} \in \mathbf{R}^{6 \times 6}, \mathbf{K}_{p}, \mathbf{K}_{V}$ are symmetric positive definite matrices,

$$
\mathbf{S}=\operatorname{diag}\left(\mathbf{S}_{i}\right) \in \mathbf{R}^{6 \times 6}, \mathbf{S}_{i}>0, \mathbf{K}_{p}+\mathbf{S} \mathbf{K}_{V}>0,\left[\begin{array}{cc}
\mathbf{S} \mathbf{K}_{p} & \mathbf{0} \\
\mathbf{0} & \mathbf{K}_{V}
\end{array}\right]>0, \tilde{\mathbf{q}}_{u}^{\prime}=\tilde{\mathbf{q}}_{u}-\delta \mathbf{q}_{u}, \dot{\tilde{\mathbf{q}}}_{u}^{\prime}=\dot{\tilde{\mathbf{q}}}_{u}-\delta \dot{\mathbf{q}}_{u},
$$

and $\delta \mathbf{q}_{u}, \delta \dot{\mathbf{q}}_{u}$ are the uncertainty vectors due to measured or estimated 6 DOF position and velocity errors, respectively.

$$
\boldsymbol{\varphi}(\cdot)=\mathbf{h}_{1}(\cdot)+\mathbf{h}_{2}(\cdot)+\mathbf{h}_{3}(\cdot)-\delta \mathbf{J}^{T} \mathbf{J}_{e}^{-T} \mathbf{v} \text {, and }\|\boldsymbol{\varphi}(\cdot)\| \leq \rho_{1}+k_{1}\|\mathbf{v}\|
$$

where

$$
\begin{gathered}
\mathbf{h}_{1}(\cdot)=-\delta \mathbf{M}(\cdot) \ddot{\mathbf{q}}_{u_{d}}-\delta \mathbf{C}(\cdot) \dot{\mathbf{q}}_{u_{d}}-\delta \mathbf{G}(\cdot)-\mathbf{J}_{e}^{T}(\cdot) \delta \mathbf{f}_{f}-\mathbf{M}(\cdot) \delta \ddot{\mathbf{q}}_{u}-\mathbf{C}(\cdot) \delta \dot{\mathbf{q}}_{u}, \\
\mathbf{h}_{2}(\cdot)=\delta \mathbf{M}(\cdot) \mathbf{S} \dot{\tilde{\mathbf{q}}}_{u}+\delta \mathbf{C}(\cdot) \mathbf{S} \tilde{\mathbf{q}}_{u}, \mathbf{h}_{3}(\cdot)=-\delta \mathbf{J}^{T} \mathbf{f}_{e q}(\cdot)+\delta \mathbf{J}^{T} \mathbf{f}_{f}, \text { and }\left\|\mathbf{h}_{1}(\cdot)+\mathbf{h}_{2}(\cdot)+\mathbf{h}_{3}(\cdot)\right\| \leq \rho_{1}(\cdot)<\rho_{2}(\cdot) \\
\mathbf{f}=\mathbf{f}_{e q}+\mathbf{J}_{e}^{-T} \mathbf{v}, \text { and } \mathbf{v}= \begin{cases}-\frac{\rho_{2}(\mathbf{e})}{1-k_{1}} \frac{\mathbf{w}}{\|\mathbf{w}\|^{\prime}}, \quad \text { if }\|\mathbf{w}\| \geq \varepsilon \\
-\frac{\rho_{2}(\mathbf{e})}{1-k_{1} \frac{\mathbf{w}}{\varepsilon},} \quad \text { if }\|\mathbf{w}\|<\varepsilon\end{cases}
\end{gathered}
$$

where $\mathbf{w}(\cdot)=\left(\dot{\tilde{\mathbf{q}}}_{U}^{\prime}+\mathbf{S} \tilde{\mathbf{q}}_{U}^{\prime}\right) \rho_{2}(\cdot)$.

Proof. Consider the following Lyapunov candidate function:

$$
V=\frac{1}{2}\left(\dot{\tilde{\mathbf{q}}}_{u}^{\prime}+\mathbf{S} \tilde{\mathbf{q}}_{u}^{\prime}\right)^{T} \mathbf{M}\left(\dot{\tilde{\mathbf{q}}}_{U}^{\prime}+\mathbf{S} \tilde{\mathbf{q}}_{u}^{\prime}\right)+\frac{1}{2} \tilde{\mathbf{q}}_{u}^{T}\left(\mathbf{K}_{P}+\mathbf{S} \mathbf{K}_{V}\right) \tilde{\mathbf{q}}_{u}^{\prime} .
$$


If the Lyapunov candidate function is chosen with real 6 DOF state (not estimated 6 DOF system state), then the practical stability cannot be rigorously proved since there exists unmatched condition caused by the bounded value in the assumption 5 . Therefore, the Lyapunov candidate function is selected as (11) on the assumption 5. The positive definite and decrescent property of this candidate function was presented in Kim et al. (2000). As a consequence, there exist constants $\gamma_{1}, \gamma_{2}>0$ such that $\gamma_{1}\|\mathbf{e}\|^{2} \leq V \leq \gamma_{2}\|\mathbf{e}\|^{2}$. With additional measurement or estimation error and the assumption 6 , the system dynamics (1) can be rearranged into

$$
\mathbf{M}(\cdot) \ddot{\tilde{\mathbf{q}}}_{U}^{\prime}+\mathbf{C}(\cdot) \dot{\tilde{\mathbf{q}}}_{U}^{\prime}=-\mathbf{M}_{0} \ddot{\mathbf{q}}_{u_{d}}-\mathbf{C}_{0} \dot{\mathbf{q}}_{u_{d}}-\mathbf{G}_{0}+\mathbf{J}_{e}^{T} \mathbf{f}-\mathbf{J}_{e}{ }^{T} \hat{\mathbf{f}}_{f}-\delta \mathbf{J}^{T} \mathbf{f}+\delta \mathbf{J}^{T} \mathbf{f}_{f}+\mathbf{h}_{1}(\cdot) .
$$

If the assumptions 1-4, and 6, the skew symmetric property of $\dot{\mathbf{M}}-2 \mathbf{C}$, and control input (10) are considered, then the derivative of the Lyapunov candidate function (11) becomes after mathematical manipulations

$$
\dot{V} \leq-\gamma_{3}\|\mathbf{e}\|^{2}+\left(\dot{\tilde{\mathbf{q}}}_{U}^{\prime}+\mathbf{S} \tilde{\mathbf{q}}_{U}^{\prime}\right)^{T} \mathbf{v}+\left\|\dot{\tilde{\mathbf{q}}}_{U}^{\prime}+\mathbf{S} \tilde{\mathbf{q}}_{u}^{\prime}\right\| \rho_{1}+k_{1}\left\|\dot{\tilde{\mathbf{q}}}_{U}^{\prime}+\mathbf{S} \tilde{\mathbf{q}}_{u}^{\prime}\right\| \cdot\|\mathbf{v}\|,
$$

where $\gamma_{3}=\lambda_{\min }\left(\left[\begin{array}{cc}\mathbf{S} \mathbf{K}_{P} & \mathbf{0} \\ \mathbf{0} & \mathbf{K}_{V}\end{array}\right]\right)$.

In the case $\|\mathbf{w}\| \geq \varepsilon$, (13) with (9) and (10) can be further reduced to yield

$$
\dot{V} \leq-\gamma_{3}\|\mathbf{e}\|^{2} .
$$

In the case $\|\mathbf{w}\|<\varepsilon_{2},(13)$ can be simplified to

$$
\dot{V} \leq-\gamma_{3}\|\mathbf{e}\|^{2}+\frac{\varepsilon}{4}
$$

The details of the derivation of (14) and (15) are shown in Khalil (1996).

Subsequently, $\varepsilon$ and $\mu(\varepsilon)$ are chosen such that $\varepsilon<2 \gamma_{3} \cdot \gamma_{2}^{-1} \cdot \gamma_{1} \cdot r^{2}$ and $\mu(\varepsilon)=\sqrt{\gamma_{3}^{-1}(\varepsilon / 4)+h}$ for $h>0$,

$$
\dot{V} \leq-\gamma_{3}\|\mathbf{e}\|^{2}+\frac{\varepsilon}{4}<0, \forall \mu(\varepsilon) \leq\|\mathbf{e}\|<r .
$$

Therefore, for any given $\varepsilon$, if $\mu(\varepsilon)<\left\|\mathbf{e}\left(t_{0}\right)\right\|<\gamma_{2}^{-1}\left(\gamma_{1}(r)\right)$, then $\dot{V}$ is strictly negative, which implies that there exists a finite time $t_{1}$ such that

$$
t_{1} \leq t_{0}+\frac{1}{\gamma_{3} h}\left\{\gamma_{2}\left\|\mathbf{e}\left(t_{0}\right)\right\|^{2}-\gamma_{1}\left(\frac{\varepsilon}{4 \gamma_{3}}+h\right)\right\},
$$

and the state stays in the set $\mathbf{D}_{\mu}=\{\mathbf{e}:\|\mathbf{e}\| \leq \mu(\varepsilon)\}$ after time $t_{1}$ (Canudus de Wit, Siciliano, \& Bastin, 1996). As a result, the system response is uniformly ultimately bounded, which implies practical stability via the controller (10). 


\section{Experiments}

In this section, the proposed task space based robust nonlinear control strategy is experimentally investigated for a 6 DOF parallel manipulator, which compares to the nonlinear control with the estimators of the system state and friction in a task space and a PID control with the system state and friction estimators in a joint space. In Fig. 2, the control block diagrams displays the implementation of the task space based robust nonlinear control strategy proposed in the previous section and another two control laws, namely, task space based nonlinear control and joint space based PID control which both treat the estimates of the system state and friction.

Fig. 3 shows the experimental apparatus of motion control system with the embedded control structure in Fig. 2, which consists of 1) Six electrical cylinders (ETS32-B08PZ20CMA150-A, Parker Inc.), 2) Control systems (Pentium III 800 PC-based system), 3) Motor amplifiers (OEM-570T, Compumotor Inc.), 4) D/A board for actuators (AT-A0-6/10, NI Corp.), 5) Encoder board (AT6450, Parker Inc.), and 6) 12bit A/D and D/A converter (LAB$\mathrm{PC}+$, NI Corp.). The sampling time for the control system is $3 \mathrm{msec}$. Rate transducer (RT020820-1, Humphrey Inc.) is applied to investigate the indirect state estimation performance as well. Table 1 describes the parameter values of the parallel manipulator. The experimental results are evaluated in the following procedure. First, the indirect method for the acquisition of the estimated 6 DOF data is examined through the results from the alpha-beta tracker with forward kinematic solution and the measured gyro signals, respectively. Second, the performance of the equivalent friction estimator is evaluated by comparison between the independently measured data (Park, 1999) and the estimates. Finally, the control performance of the proposed robust nonlinear control law (10) in task space is compared to the task space based nonlinear control law (8) and the joint space based PID control with the estimators for the system state and friction.

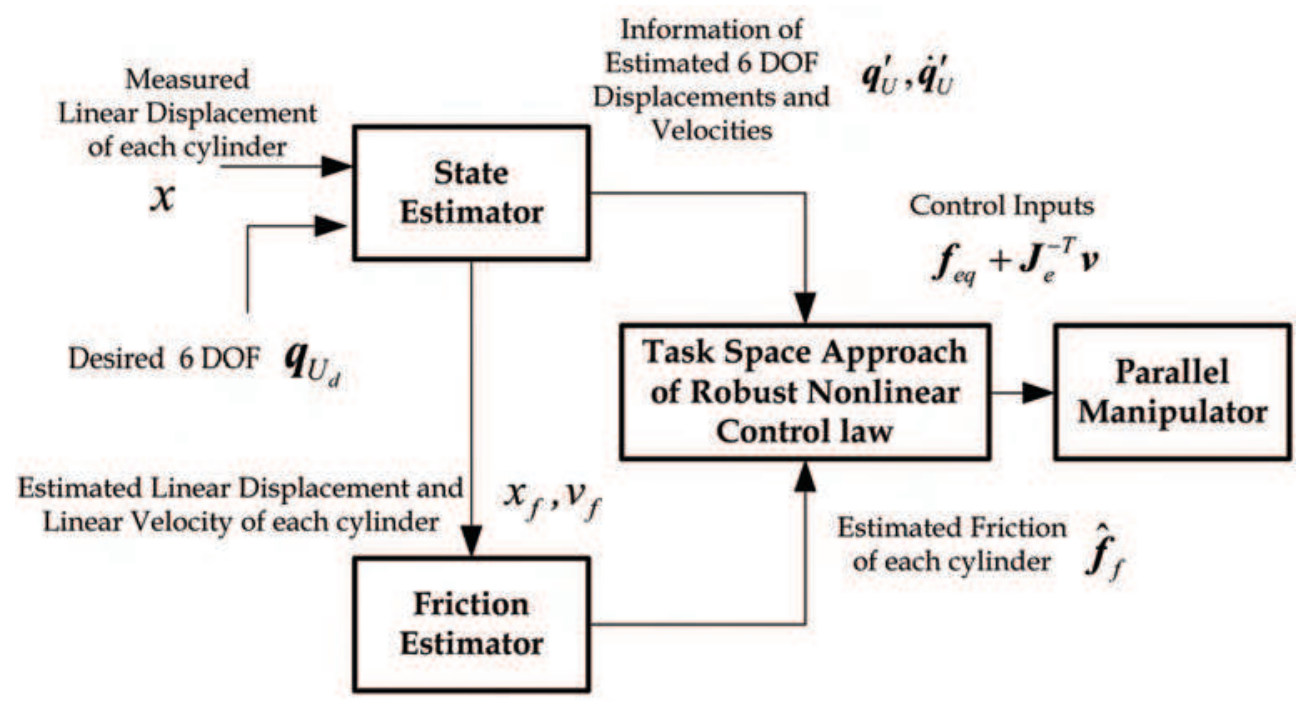

Fig. 2. Control block diagrams (a) Task space based robust nonlinear control scheme with the system state and friction estimators 
Measured

Linear Displacement

of each cylinder
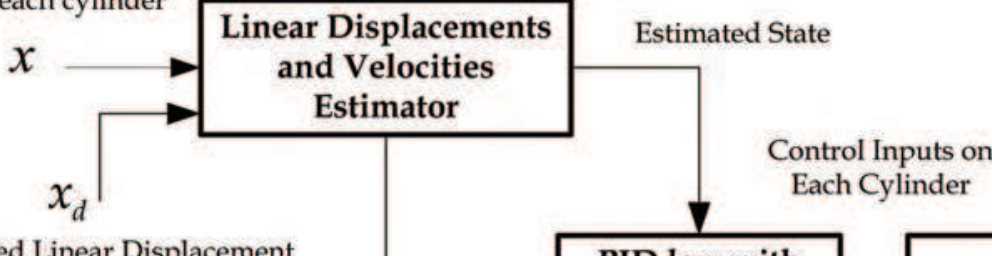

Desired Linear Displacement

of each cylinder

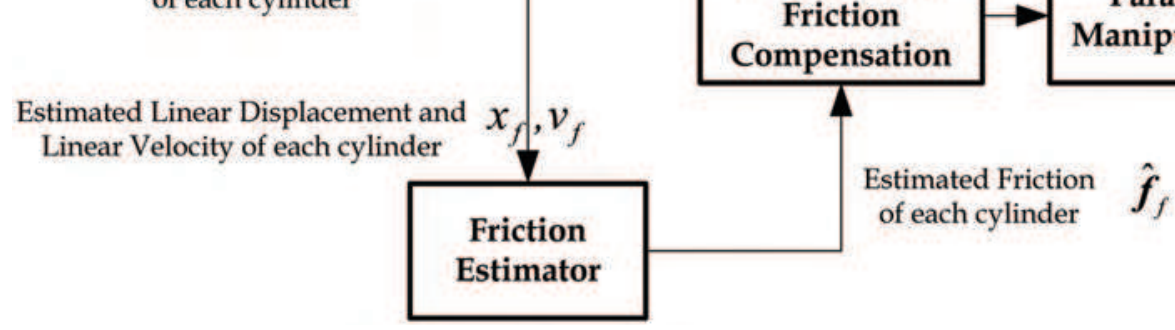

Fig. 2. Control block diagrams (b) Joint space based PID control with the estimators of the system state and friction

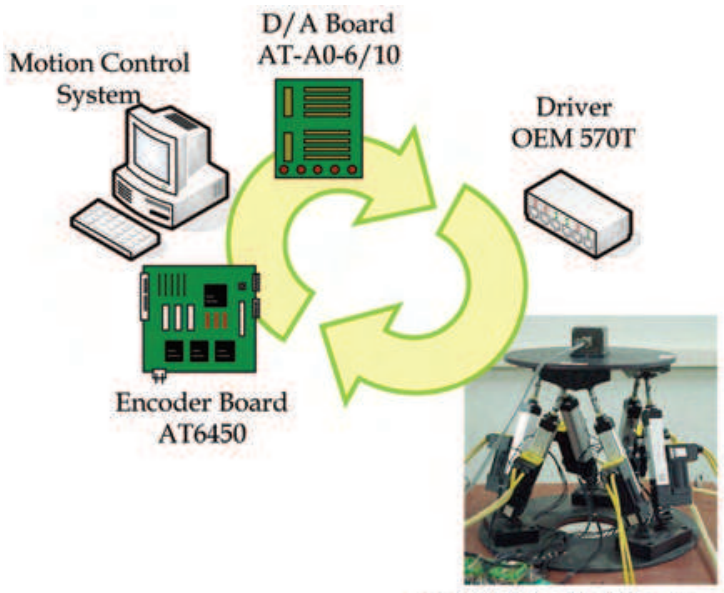

6 DOF Parallel Manipulator

Fig. 3. Experimental apparatus of motion control system

\begin{tabular}{cccc}
\hline Parameter & Description & Value & Unit \\
\hline \hline$l_{\min }, l_{\max }$ & Min./Max. Stroke of Cylinder & $0.365 / 0.51$ & {$[\mathrm{~m}]$} \\
\hline$m_{U}$ & Mass of Upper Plate & 24.0 & {$[\mathrm{Kg}]$} \\
\hline$I_{x x}, I_{y y}, I_{z z}$ & Moment of Inertia of Upper Plate & $0.4315,0.4316,0.6111$ & {$\left[\mathrm{Kg} \cdot \mathrm{m}^{2}\right]$} \\
\hline$r_{L}, r_{U}$ & Radius of Lower Plate/Upper Plate & $0.24 / 0.16$ & {$[\mathrm{~m}]$} \\
\hline
\end{tabular}

Table 1. Parameter values of a 6 DOF parallel manipulator 


\subsection{State estimator}

In this sub-section, the performance of the numerical method and the alpha-beta tracker are investigated to confirm the estimated system state to be feasible prior to the application for the task space based control approach. The sensing and estimation procedure is enumerated as follows;

1) Measure the length of each cylinder.

2) Use the alpha-beta tracker for each length signal

3) Apply the numerical method to obtain a forward kinematic solution

4) Use alpha-beta tracker to acquire the derivatives of 6 DOF positional state from the forward kinematic solution

Firstly, the measured cylinder lengths are compared to the inverse kinematic solution based on the $6 \mathrm{DOF}$ estimates from the Newton-Raphson numerical method (tolerance $10^{-7}$ ) as in Fig. 4 . The result shows less than $0.1 \%$ errors (normal length $435 \mathrm{~mm}$ ) to a multi-directional sinusoidal inputs (roll $\left(2.0^{\circ} / 1.0 \mathrm{~Hz}\right)$, pitch $\left(5.0^{\circ} / 0.5 \mathrm{~Hz}\right)$, yaw $\left(2.5^{\circ} / 1.0 \mathrm{~Hz}\right.$ ) and heave $(5.0 \mathrm{~mm} / 0.5 \mathrm{~Hz}))$, which verifies that the assumption 4 is satisfied since $k_{1}$ is less than $0.1 \mathrm{in}$ the case of the intended $\pm 5 \%$ uncertainty in each cylinder length. The installed rate transducer (RT02-0820-1, Humphrey Inc.) as a sensor providing a base line has checked the fidelity of the 6 DOF estimator through the comparison between the estimated and measured rotational velocities of angular motions.

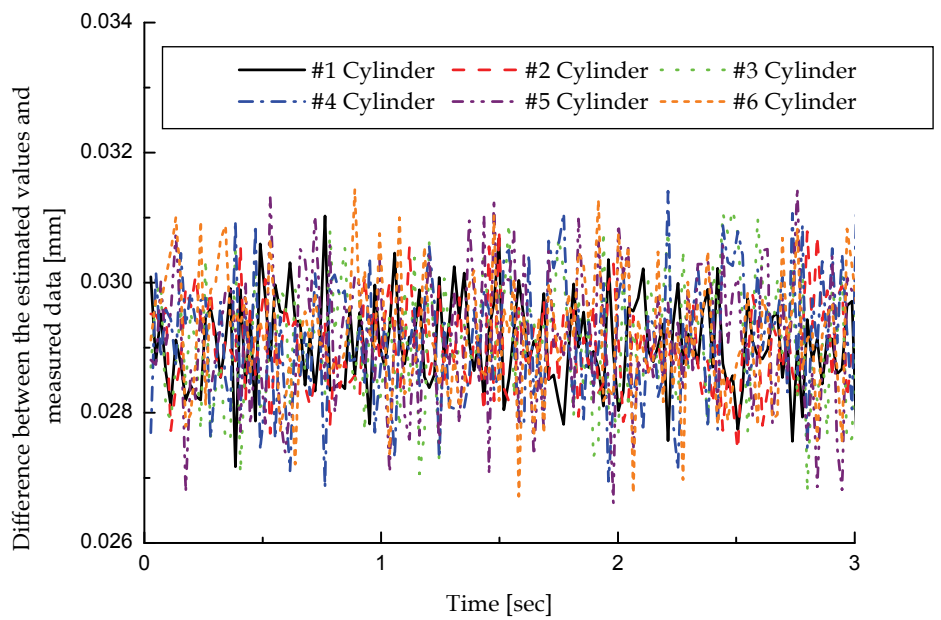

Fig. 4. Errors between measured lengths and inverse kinematic solutions from the estimated 6 DOF positional data based on the numerical method and the alpha-beta tracker

In Fig. 5, the comparisons between the rate transducer readings and the estimated angular velocities to sinusoidal position inputs (Roll: $2.0^{\circ} / 1.0 \mathrm{~Hz}$, Pitch: $5.0^{\circ} / 0.5 \mathrm{~Hz}$ ), which gives fidelity that the estimation scheme truly yields the derivatives of motion signals without complicated calculation and the assumption 5 is feasible. 

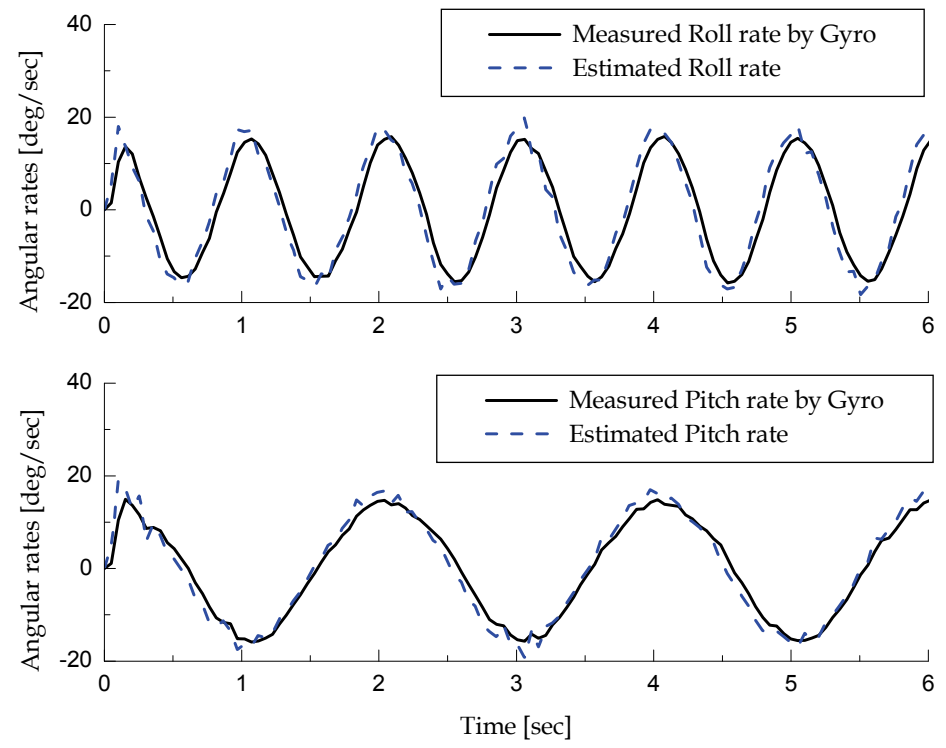

Fig. 5. The measured data and the estimated signals by the alpha-beta tracker to sinusoidal inputs (Roll: $2.0^{\circ} / 1.0 \mathrm{~Hz}$, Pitch: $5.0^{\circ} / 0.5 \mathrm{~Hz}$ )

\subsection{Friction estimator}

This subsection describes the friction estimator proposed to reject the frictional disturbance for the enhanced control performance. As mentioned in Section 3, the excessive or deficient feed-forward friction compensation to step input under uncertain frictional disturbance makes an oscillatory or a sticking steady state, respectively. Fig. 6 shows the example of the phenomenon when incongruent feed-forward friction compensation with independently measured value (Park, 1999) is applied to the same system (the result can be compare to that of Fig. 8 (a) in subsection 5.3 later).

Fig. 7 presents that comparison of errors between PID control with friction estimator and PID control without friction compensator to a roll input (Sine: $5^{\circ} / 0.5 \mathrm{~Hz}$ ), which explains that the friction compensator is truly required. The figure also shows that the good friction estimation result of the 3rd cylinder (other cylinders have similar results) through PID control with the friction estimator and control performance becoming better as the time increases. The gains $k_{f}$ and $\mu_{f}$ are 10.0 and 1.5, respectively in this estimator. It should be noted that the independently measured friction property of this parallel system (Park, 1999) may depends on load condition, lubrication condition, temperature, even time, etc. In the proposed robust nonlinear control, the difference between the bounded real friction and the estimated friction is considered as the element of the system uncertainty in (9). 

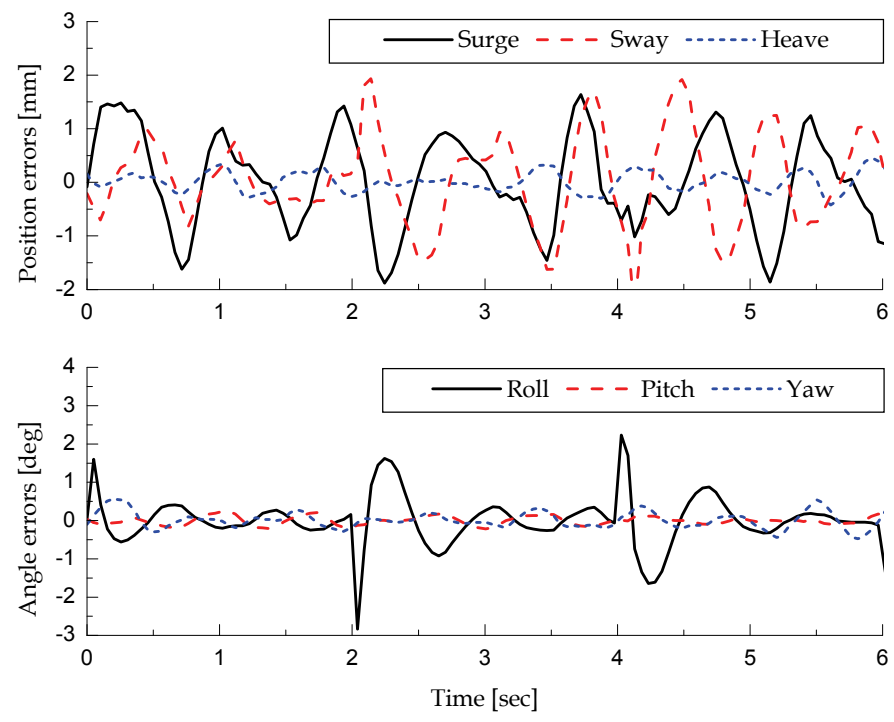

Fig. 6. Influence of the feed-forward friction compensation with PID control to a step input (Roll: $5.0^{\circ} / 0.25 \mathrm{~Hz}$ )
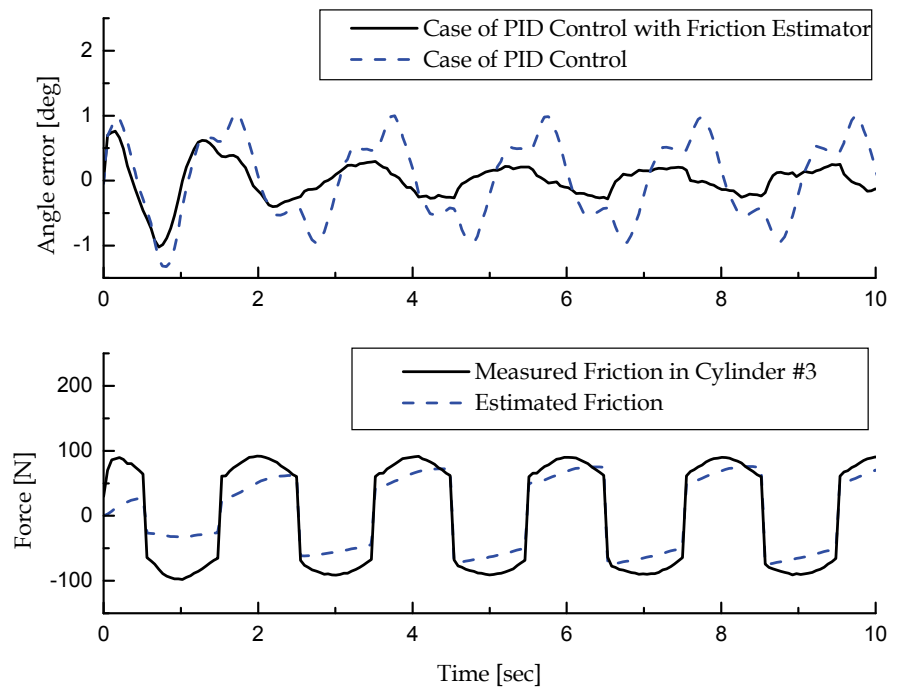

Fig. 7. Effectiveness of a friction compensator with PID control to a sinusoidal input (Roll: $\left.5.0^{\circ} / 0.5 \mathrm{~Hz}\right)$ 


\subsection{Regulating and tracking performances}

In this subsection, the control performance of the proposed task space based robust nonlinear control law with the estimators for the system state and friction (hereafter this control law is named TRNCE) is presented. As unbiased benchmarking controllers, task space based nonlinear control law with the system state and friction estimation method (hereafter the control law is called TNCE) and joint space based PID control law with the estimators for the system state and friction (hereafter the control law is PIDE) are employed. It should be noted that TNCE (8) is similar to a task space based PD controller and handles the perfectly known nonlinearities. On the other hand, the TRNCE (10) deals with the uncertainties of system parameters and frictions additionally. The experimentally tuned PID control gains $K_{P_{-} \text {gain }}, K_{I_{-} \text {gain }}, K_{D_{-} \text {gain }}$ are 100, 800, and 20, respectively, which result in smaller steady state errors than those by the gains in Park (1999). The control gains for the TRNCE and TNCE are:

$$
\begin{gathered}
\mathbf{K}_{P}=1 \times 10^{5} \cdot\left[\begin{array}{cccccc}
0.456 & 0 & 0 & 0 & 0.0234 & 0 \\
0 & 0.456 & 0 & -0.01404 & 0 & 0 \\
0 & 0 & 3.75 & 0 & 0 & 0 \\
0 & -0.01404 & 0 & 0.0312 & 0 & 0 \\
0.0234 & 0 & 0 & 0 & 0.052 & 0 \\
0 & 0 & 0 & 0 & 0 & 0.0208
\end{array}\right], \\
\mathbf{K}_{V}=1 \times 10^{4} \cdot\left[\begin{array}{cccccc}
0.06 & 0 & 0 & 0 & 0.009 & 0 \\
0 & 0.12 & 0 & -0.009 & 0 & 0 \\
0 & 0 & 1.16 & 0 & 0 & 0 \\
0 & -0.009 & 0 & 0.02 & 0 & 0 \\
0.009 & 0 & 0 & 0 & 0.02 & 0 \\
0 & 0 & 0 & 0 & 0 & 0.0027
\end{array}\right],
\end{gathered}
$$

$\mathbf{S}=10 \cdot \mathbf{I}, \varepsilon=2.0$, and $k_{1}=0.1$.

It is further assumed that there exist such system uncertainties as $5 \%$ in inertia, $5 \%$ in gravity force and $1 \%$ in Jacobian. The gain matrices chosen above can be easily confirmed the positive definiteness condition in Theorem 8. The TRNCE gains seem much higher than those of PIDE. However, it comes from that the TRNCE calculates the desired force from the gain matrices and Jacobian, while the PIDE produces just control input calculated by the position errors and estimated friction.

Firstly, the regulation performance is investigated. Fig. 8 shows that the nonlinear approaches (TRNCE and TNCE) have superior overall regulating performance to a step input (Roll: $5^{\circ} / 0.25 \mathrm{~Hz}$ ) than the PIDE. With a view point of pseudo- steady-state error to roll motion input, TRNCE shows $\pm 0.3^{\circ}$ of error bound; on the other hand, the PIDE shows $\pm 0.1^{\circ}$ of superior error bound even though there exists $16 \%$ overshoot in the transient response. However, large and oscillatory errors by the PIDE are observed in the other motions; the other motion errors by PIDE are twice or more those by the TRNCE. The regulating performances by TNCE show the similar to those by TRNCE. The above outcomes stem from the fact that the PIDE does not consider the sensitivity of 6 DOF 
displacements on length variation, that is, Jacobian, which results in overall performance in task space to be inferior to those of TRNCE and TNCE. Reducing the magnitude of $\varepsilon$ may give further enhancement of regulation performance. However, such an approach may degrade the control performance by chattering effect as described in Khalil (1996) due to a fast switching control input that may excite high frequency modes in the system.
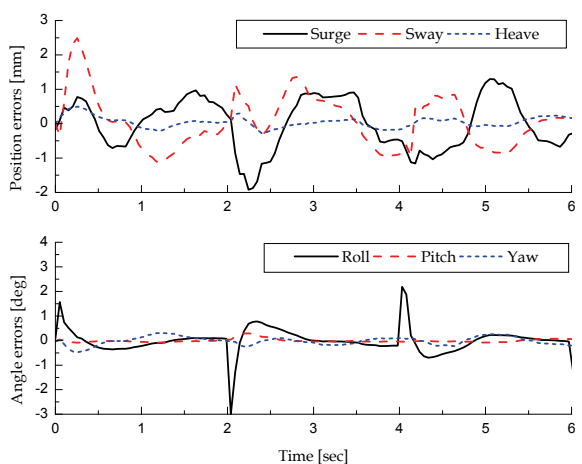

(a) PIDE
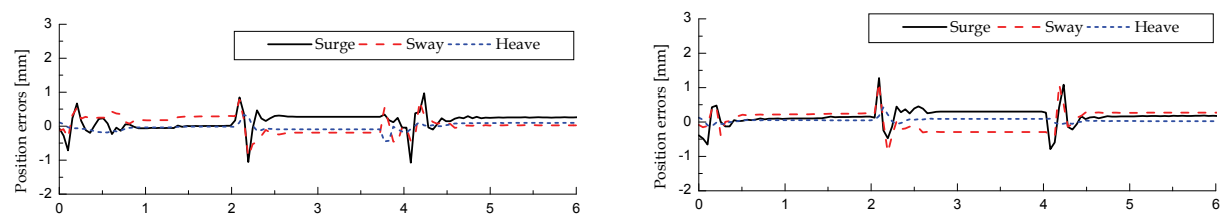

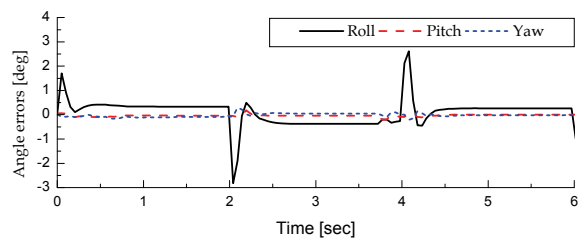

(b) TNCE

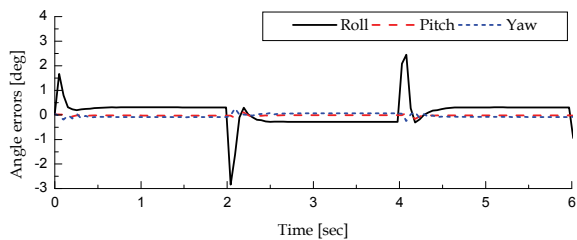

(c) TRNCE

Fig. 8. Regulating errors of 6DOF motions to a step input (Roll: $5.0^{\circ} / 0.25 \mathrm{~Hz}$ )

Tracking errors to a sinusoidal input of roll motion $\left(5.0^{\circ} / 0.5 \mathrm{~Hz}\right)$ are examined as well (not in this paper). In a steady state, the translation error bounds of the TRNCE are smaller than $+0.41 /-0.4 \mathrm{~mm}$, those of the TNCE are smaller than $+0.45 /-0.5 \mathrm{~mm}$, while those of the PIDE are larger than $+0.8 /-1.1 \mathrm{~mm}$. All the rotational errors of the TRNCE are bounded below $+0.28^{\circ} /-0.31^{\circ}$, while the maximum errors of the TNCE are stayed at $+0.34^{\circ} /-0.49^{\circ}$ and maximum errors of the PIDE are smaller than $\pm 0.29^{\circ}$. With a viewpoint in only comparison of the min/max steady state error in a roll direction, the PIDE shows the slightly better performance. However, the simple comparisons of maximum and minimum error values 
cannot represent the overall tracking performance. Therefore, the RMS (root mean square) values in the errors are investigated to confirm the comprehensive tracking performance. If each RMS value of 6 DOF motion errors by PIDE is defined as $100 \%$, then each RMS value of motion errors along six directions (surge, sway, heave, roll, pitch, and yaw) is $40 \%, 34 \%$, $39 \%, 94 \%, 91 \%$, and $62 \%$ for TNCE, and $31 \%, 34 \%, 37 \%, 72 \%, 90 \%$, and $35 \%$ for TRNCE, respectively. The RMS values of errors show that nonlinear control laws designed in task space are superior to the PIDE. Furthermore, the TRNCE exhibits the more excellent control performance than the TNCE by the RMS values of errors and the comparison of each maximum value, which result from the reflection of the system uncertainties.
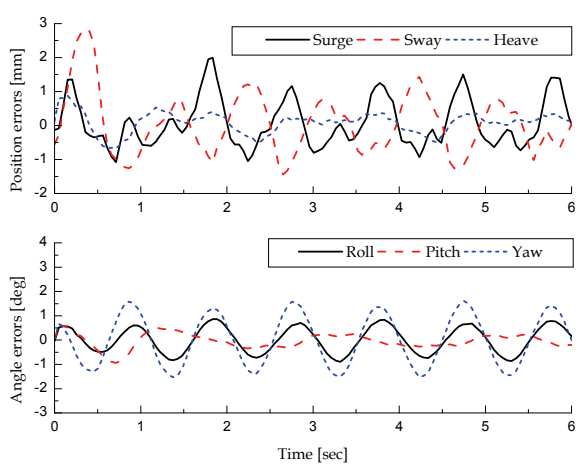

(a) PIDE
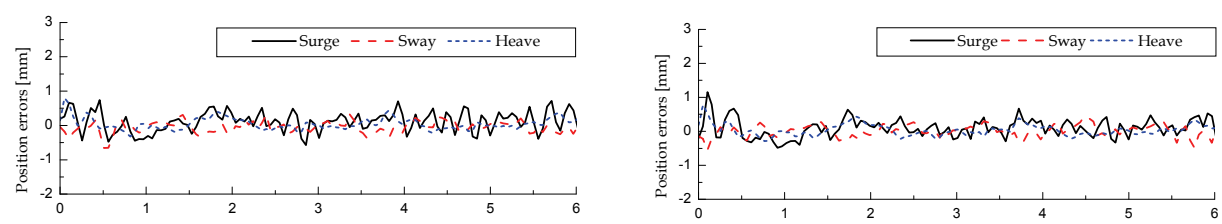

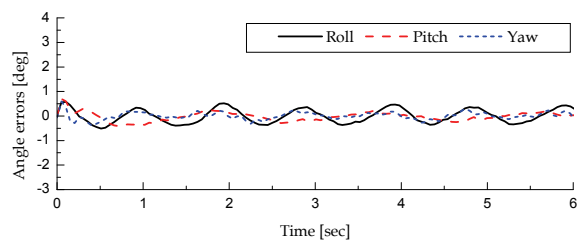

(b) TNCE

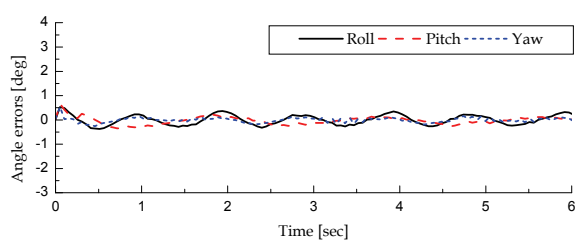

(c) TRNCE

Fig. 9. Tracking errors of 6DOF motions to multi-directional sinusoidal inputs (Roll: $2.0^{\circ} / 1.0$ Hz, Pitch: 5.0\%/0.5 Hz, Yaw: 2.5\%/1.0 Hz, and Heave: 5.0 mm/0.5 Hz)

Fig. 9 presents tracking errors to multi-directional sinusoidal inputs (Roll: $2.0^{\circ} / 1.0 \mathrm{~Hz}$, Pitch: $5.0^{\circ} / 0.5 \mathrm{~Hz}$, Yaw: $2.5^{\circ} / 1.0 \mathrm{~Hz}$, and Heave: $5.0 \mathrm{~mm} / 0.5 \mathrm{~Hz}$ ). The TRNCE and TNCE show the remarkable tracking performances superior to those of the PIDE in all 6 DOF directions which is similar in performance tendency to the previous case. The superb performances 
through the TRNCE and TNCE result from the task space based designs and cancellation of nonlinearities (the inertia force for a given acceleration, the gravitational force, the Coriolis and centrifugal forces). The translation errors of the TRNCE are bounded between $+0.77 \mathrm{~mm}$ and $-0.48 \mathrm{~mm}$, those of the TNCE lie between $+0.76 \mathrm{~mm}$ and $-0.52 \mathrm{~mm}$, while those of the PIDE exceed $\pm 1.5 \mathrm{~mm}$ in a steady state. All the rotational error bounds of the TRNCE lie within $\pm 0.35^{\circ}$, maximum error of the TNCE are bounded below $\pm 0.45^{\circ}$, while those of the PIDE exceeds $\pm 1.5^{\circ}$. The RMS (root mean square) values in the errors are also investigated to confirm the comprehensive tracking performance. In the case that each RMS value of the 6 DOF motion errors is also defined as $100 \%$ by PIDE, each RMS (root mean square) value of the motion errors along six directions (surge, sway, heave, roll, pitch, and yaw) is $45 \%, 23 \%$, $58 \%, 51 \%, 66 \%$, and $13 \%$ for TNCE and $38 \%, 23 \%, 56 \%, 36 \%, 57 \%$, and $9 \%$ for TRNCE, respectively. There exists the difference in control performance between the TRNCE and the TNCE, which stems from the additional robust control input considering the system uncertainties. Consequently, it is shown that the TRNCE excels the TNCE and the PIDE in terms of control performances to the multi-directional sinusoidal inputs with high frequency component.

\section{Conclusion}

This paper proposes and implements the task space approach of a robust nonlinear control with the system state and friction estimation methodologies for the parallel manipulator which is a representative multi-input \& multi-output nonlinear system with uncertainties. In order to implement the proposed robust nonlinear control law, the indirect 6 DOF system state estimator is firstly employed and confirmed the outstanding effects experimentally. The indirect system state estimation scheme consists of Newton-Raphson method and the alpha-beta tracker algorithm, which is simple route and readily applicable to a real system instead of a costly 6 DOF sensor or a model-based nonlinear state observer with the actuator length measurements. Secondly, the Friedland-Park friction observer is applied as the equivalent friction estimator in joint space which provides the friction estimates to attenuate uncertain frictional disturbance. The suitability of this friction estimation approach is experimentally confirmed as well. Finally, the control performances of the proposed task space based robust nonlinear control law equipped with the estimators of system state and the friction are experimentally evaluated. With viewpoints of regulating and tracking, the remarkable control results to several inputs are shown under system nonlinearity, parameter uncertainties, uncertain friction property, etc. In addition to those, the experimental results shows that the proposed robust nonlinear control scheme in task space surpasses the nonlinear task space control with the estimators and the joint space based PID control with the estimators, which reveal its availability to the practical applications like a robotic system or machine-tool required the task space based control scheme for a precision control performance.

\section{References}

Amstrong-Hélouvry; B., Dupont, P. \& Canudas de Wit, C. (1994). A Survey of Models, Analysis Tools and Compensation Methods for the Control of Machines with Friction. Automatica, Vol. 30, No. 7, pp. 1083-1138. 
Barmish, B. R.; Corless, M. J. \& Leitmann, G. (1983). A New Class of Stabilizing Controllers for Uncertain Dynamical Systems. SIAM Journal of Control and Optimization, Vol. 21, pp. 246-255.

Canudus de Wit, C.; Siciliano, B. \& Bastin, G. (1996). Theory of Robot Control, Springer, Berlin. Corless, M. J. \& Leitmann, G. (1981). Continuous State Feedback Guaranteeing Uniform Ultimate Boundedness for Uncertain Dynamic Systems. IEEE Transactions on Automatic Control, Vol. 26, pp. 153-158.

Dasgupta, B. \& Mruthyunjaya, T. S. (1998). Closed-Form Dynamic Equations of the General Stewart Platform through the Newton-Euler Approach. Mechanism and Machine Theory, Vol. 33, pp. 993-1012.

Dieudonne, J. E.; Parrish, R. V. \& Bardusch, R. E. (1972). An Actuator Extension Transformation for a Motion Simulator and an Inverse Transformation applying Newton-Raphson Method. NASA Technical Report D-7067.

Friedland, B. (1973). Optimum Steady-State Position and Velocity Estimation Using Sampled Position Data, IEEE Transactions on Aerospace and Electronic Systems, AESVol. 9, No. 6, pp. 906-911.

Friedland, B. \& Park, Y. J. (1992). On Adaptive Friction Compensation. IEEE Transactions on Automatic Control, Vol. 37, No. 10, pp. 1609-1612.

Hahn, W. (1967). Stability of Motion, Springer, New York.

Honegger, M.; Brega, R. \& Schweitzer, G. (2000). Application of a Nonlinear Adaptive Controller to a 6 dof Parallel Manipulator. In Proceeding of the 2000 IEEE International Conference on Robotics and Automation, pp. 1930-1935, San Francisco, April, 2000, CA., USA.

Kang, J. Y.; Kim, D. H. \& Lee, K. I. (1996) Robust Tracking Control of Stewart Platform. In Proceedings of the 35th Conference of Decision and Control, pp. 3014-3019, Kobe, December, 1996, Japan.

Kang, J. Y.; Kim, D. H. \& Lee, K. I. (1998). Robust Estimator Design for Forward Kinematics Solution of a Stewart Platform. Journal of Robotic Systems, Vol. 15, Issue 1, pp. 30-42.

Khalil, H. K. (1996). Nonlinear Systems, 2nd ed.,Prentice-Hall, New Jersey.

Kim, D. H.; Kang, J. Y. \& Lee, K. I. (2000). Robust Tracking Control Design for a 6 DOF Parallel Manipulator. Journal of Robotic Systems, Vol. 17, Issue 10, pp. 527-547.

Lewis, F. (1986). Optimal Estimation with an Introduction to Stochastic Control Theory, John Wiley and Sons, Inc, USA.

Merlet, J. P. (2000). Parallel Robots, Kluwer Academic Publisher, Netherlands.

Nguyen, C. C.; Antrazi, S., Zhou, Z. L. \& Campbell, C. (1993). Adaptive Control of a Stewart Platform-Based Manipulator. Journal of Robotic Systems, Vol. 10, No. 5, pp.657-687

Panteley, E.; Ortega, R. \& Gafvert, M. (1998). An Adaptive friction compensator for global tracking in robot manipulators, Systems \& Control Letters, Vol. 33, Issue 5, pp. 307313.

Park, C. G. (1999). Analysis of Dynamics including Leg Inertia and Robust Controller Design for a Stewart Platform, Ph. D. thesis, Seoul National University, Korea.

Radcliffe, C. J. \& Southward, S. C. (1990). A Property of Stick-Slip Friction Models which Promotes Limit Cycle Generation. In Proceedings on American Control Conference, pp. 1198-1203, May, 1990, San Diego, USA. 
Sirouspour, M. R. \& Salcudean, S. E. (2001). Nonlinear Control of Hydraulic Robots, IEEE Transactions on Robotics and Automation, Vol. 17, No. 2, pp. 173-182.

Spong, M. W. \& Vidyasagar, M. (1989). Robot Dynamics and Control, John Wiley \& Sons, Inc.

Ting, Y.; Chen, Y. S. \& Wang, S. M. (1999). Task-space Control Algorithm for Stewart Platform. In Proceedings of the 38th Conference on Decision and Control, pp. 3857-3862, December, 1999, Phoenix, Arizona, USA. 


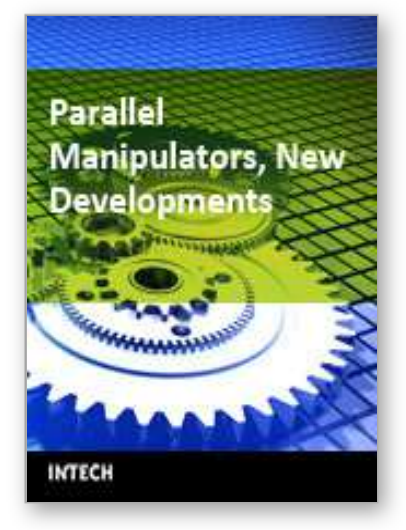

\author{
Parallel Manipulators, New Developments \\ Edited by Jee-Hwan Ryu
}

ISBN 978-3-902613-20-2

Hard cover, 498 pages

Publisher I-Tech Education and Publishing

Published online 01, April, 2008

Published in print edition April, 2008

Parallel manipulators are characterized as having closed-loop kinematic chains. Compared to serial manipulators, which have open-ended structure, parallel manipulators have many advantages in terms of accuracy, rigidity and ability to manipulate heavy loads. Therefore, they have been getting many attentions in astronomy to flight simulators and especially in machine-tool industries. The aim of this book is to provide an overview of the state-of-art, to present new ideas, original results and practical experiences in parallel manipulators. This book mainly introduces advanced kinematic and dynamic analysis methods and cutting edge control technologies for parallel manipulators. Even though this book only contains several samples of research activities on parallel manipulators, I believe this book can give an idea to the reader about what has been done in the field recently, and what kind of open problems are in this area.

\title{
How to reference
}

In order to correctly reference this scholarly work, feel free to copy and paste the following:

Hag Seong Kim (2008). Task Space Approach of Robust Nonlinear Control for a 6 DOF Parallel Manipulator, Parallel Manipulators, New Developments, Jee-Hwan Ryu (Ed.), ISBN: 978-3-902613-20-2, InTech, Available from:

http://www.intechopen.com/books/parallel_manipulators_new_developments/task_space_approach_of_robust _nonlinear_control_for_a_6_dof_parallel_manipulator

\section{INTECH}

open science | open minds

\author{
InTech Europe \\ University Campus STeP Ri \\ Slavka Krautzeka 83/A \\ 51000 Rijeka, Croatia \\ Phone: +385 (51) 770447 \\ Fax: +385 (51) 686166 \\ www.intechopen.com
}

\author{
InTech China \\ Unit 405, Office Block, Hotel Equatorial Shanghai \\ No.65, Yan An Road (West), Shanghai, 200040, China \\ 中国上海市延安西路65号上海国际贵都大饭店办公楼 405 单元 \\ Phone: +86-21-62489820 \\ Fax: +86-21-62489821
}


(C) 2008 The Author(s). Licensee IntechOpen. This chapter is distributed under the terms of the Creative Commons Attribution-NonCommercialShareAlike-3.0 License, which permits use, distribution and reproduction for non-commercial purposes, provided the original is properly cited and derivative works building on this content are distributed under the same license. 